\title{
Benchmarking Agility For Multi-legged Terrestrial Robots
}

\author{
Peter Eckert and Auke J. Ijspeert \\ Biorobotics Laboratory, EPFL, Switzerland
}

\begin{abstract}
In this paper, we present a novel and practical approach for benchmarking agility. We focus on terrestrial, multi-legged locomotion in the field of bio-inspired robotics. We define agility as the ability to perform a set of different but specific tasks executed in a fast and efficient manner. This definition is inspired by the analysis of natural role models, such as dogs and horses as well as robotic systems. An evaluation of existing benchmarks in robotics is done and taken into account in our proposed benchmark. After the general definition, the actual normalized benchmarking values are defined, and measuring methods, as well as an online database for agility score collection and distribution, are presented. To provide a baseline for agile locomotion, various videos of dog-agility competitions were analyzed and agility scores calculated where applicable. Finally, validation and implementation of the benchmark are done with different robots directly available to the authors. In conclusion, our benchmark will enable researchers not only to compare existing robots and find out strengths and weaknesses in different design approaches, but also give a tool to define new fitness functions for optimization, learning processes and future robots developments, intensifying the links between biology and technology even further.
\end{abstract}

\section{INTRODUCTION}

Agility is one of the terms that make people realize that an animal, a robot, or some other system is extraordinary in some manner. It is often associated with the speed of executing a specific task, like moving forward or turning. It is additionally used in a manifold of areas, such as business, production [1], or animal sports, but sometimes with completely different meanings. The same word contains, depending on the field of usage, different key aspects and is thus not homogeneous in its definition. But what exactly is agility then? How can it be described, quantified and what does it imply for the field of mobile robotics? One possible, mainly locomotion-related, definition is found in Wikipedia [2]:

\section{[...] Agility or nimbleness is the ability to change}

the body's position efficiently and requires the integration of isolated movement skills using a combination of balance, coordination, speed, reflexes, strength, and endurance. Agility is the ability to change the direction of the body in an efficient and effective manner [...]

This definition, although unreferenced, gives a good highlevel view of locomotion-related agility with its multitude of components. Consequently, the agility of a system or a being is hard to grasp, measure and quantify. Hints on how to draw a definition of it and build a corresponding benchmark may be taken from a great source of inspiration for technological systems, nature. Here, agility manifests in various species. Furthermore, humans strive to compare and measure themselves and their animal partners throughout various kinds of competitions highlights specific clues towards finding a solution to our benchmark related problem.

In this work, we focus on the agility definition related to the field of multi-legged, terrestrial locomotion. Covering even more areas of locomotion would surpass the framework of this paper and may be addressed in future publications. We start by presenting a concise definition of the term agility, as well as a method to quantify and compare different systems. Natural role models will be analyzed to draw out baselines (or normalization values). Creating a benchmark will enable robotics researchers and biologists to compare their research object, i.e., give new fitness functions for learning or optimization processes, identify weaknesses of their systems (mechanical or in control) as well as point towards existing role models when starting their development processes.

\section{Agility in Human- And Animal-Sports}

This section will highlight our observations towards defining and benchmarking agility when looking at human- and animalsports. Agility is not fixed to ground locomotion, but also flying, swimming, and diving. Our interest, in this publication, lies in terrestrial, multi-legged locomotion. Observations from sports are generally qualitative but will influence our benchmarking structure decisively as can be seen in the conclusion of this section.

a) Agility in Animal-Sports: In sports that are done in cooperation of human and animal, two examples of extreme agility-demonstration come to mind: dog-agility, where the name already includes the main feature of the sport, and horseshow-jumping, that also provides an impressive demonstration of control and explosive force. The core of both sports is a series of complex movements, executed with a minimal number of mistakes and completed as fast as possible. Like all sports, high amounts of energy are used by the animals, resulting in visible fatigue, also to be included in our observations.

In horse show-jumping, the animal (with the human on the back) has to perform a series of leaps over different shaped obstacles in combination with a pre-defined path, including accelerations and sharp turns. This sport demands from the horse the ability to precisely follow the commands of its rider and to explosively execute difficult jumping and turning tasks in succession of each other. Time and precision are of the essence. A scoring scheme also includes a penalty system taking into account failures in execution of any task (e.g., knocking down a rail).

Dog agility varies even more in the complexity of the tasks at hand. The dog has to follow a specific course of jumps, ramps, balancing-boards, and other obstacles as fast as possible, with particular stops to test control, making the 
perfect run even more difficult. The dog-trainer is allowed to give directional commands, as guidance. The decision on how to fulfill these is up to the dog but also influenced by lengthy and intense training beforehand. The quick and fault-minimal fulfillment of the course is taken as the grading measure of this sport.

Our observation showed that agility in animal sport is focused mainly on precision and speed. The best reference is dog-agility as the task-space is vast. It serves additionally as a guide to draw a baseline for comparison and normalization.

b) Agility in Human-Sports: [3] intensively analyzed the role of agility in human sports through a literature review of different sports scientists. The findings of their work are summarized below and concur widely with the observations we had from our animal analysis. Criteria for agility are:

1) Must involve the initiation of body movement, change of direction, or rapid acceleration or deceleration.

2) Must involve whole-body movement.

3) Involves considerable uncertainty, whether spatial or temporal.

4) Open skills only (meaning skills that do not require a pre-learned stimulus to be activated; one could say: natural behavior).

5) Involves a physical and cognitive component, such as recognition of a stimulus, reaction, or execution of physical response (the skill must be activated by recognizing its need due to outside factors, e.g., leg retraction induced by hitting an obstacle with the foot).

Agility in their opinion should incorporate the whole body with changes of direction executed in a reactive rather than a planned manner. Reactive behaviors show the bodies general readiness to cope with uncertain situations and thus react nimbly or with agility. Preplanned behavior can make use of motion patterns one would not naturally use for the task at hand, but which can give (especially in sports) the overall best performance in this specific task. They exclude preplanned skills like straight and steady running from the term agility. Some of these excluded skills, like fast forward running, might in our opinion still be valid to include in the agility definition as performing them shows excellent value for locomotion itself. Another interesting approach is presented in [4]. Here not agility in human sports per se is researched, but a benchmark for human-likeness of bipedal robots is defined. Although there is no time factor involved in the referenced work, many different tasks are defined, that the robot has to fulfill to get a good score. The idea of separating behaviors is exciting and concurs with our views on how to determine agility.

c) Conclusion from Human- and Animal-Sports: To conclude our observations of nature, there are some key aspects of locomotion that can be seen as main features to describe agility adequately and simple enough for further quantification:

1) Agility is not a single skill, but a complex set of motion patterns as well as the possibility to rapidly switch between them.

2) Ideally, reactive execution of known skills with minimal prior planning

3) Agility varies from one species to another and thus should, at least, be defined differently in terrestrial, aerial and aquatic locomotion (in case of interest in aquatic robots, please refer to [5]).

4) Precision in task execution is one of the key aspects.

5) Speed of the task execution is another key aspect.

6) Agility is related to the scale of the system or animal. Thus it should be normalized to attempt a comparison.

7) The energy-cost to execute a task should be part of benchmarking a system's agility.

With these findings in mind, we will evaluate the robotic world and its attempts to quantify agility in the field of terrestrial legged locomotion as well as propose our method.

\section{AgILITy IN LEGGED, TERRESTRIAl Robotics}

This section shows our observations towards defining and benchmarking agility when looking at it from a more technological perspective. Based on existing quantification attempts of robots claiming to be agile, we will look at generally applied metrics and draw out their essence in the conclusion of this section.

Table I: Comparison of selected quadruped robots table data adapted from [6], and extended; Froude number $\left(F R=\bar{v}^{2} /(g\right.$. h)),BL/S body-lengths/second

\begin{tabular}{llllllll} 
Robot & & $\begin{array}{l}\mathrm{m}_{\text {rob }} \\
{[\mathrm{kg}]}\end{array}$ & $\begin{array}{l}\mathrm{h}_{\text {hip }} \\
{[\mathrm{m}]}\end{array}$ & $\begin{array}{l}\mathrm{l}_{\text {rob }} \\
{[\mathrm{m}]}\end{array}$ & $\begin{array}{l}\mathrm{v}_{\text {max }} \\
{\left[\mathrm{m} \mathrm{s}^{-1}\right]}\end{array}$ & $\begin{array}{l}\mathrm{FR} / \mathrm{s} \\
{\left[\mathrm{s}^{-1}\right]}\end{array}$ \\
\hline Scout II & {$[7]$} & 20.865 & 0.323 & 0.552 & 1.3 & 0.53 & 2.4 \\
BigDog & {$[8]$} & 109 & 1 & 1.1 & 3.1 & 0.98 & 2.8 \\
ANYmal & {$[9]$} & 30 & $\approx 0.4$ & $\approx 0.5$ & 0.8 & 0.16 & 1.6 \\
StarlETH & {$[10]$} & 23 & $\approx 0.5$ & 0.5 & 0.75 & 0.12 & 1.5 \\
\hline HyQ & {$[11]$} & 91 & 0.789 & 1.0 & $\approx 2$ & 0.52 & $\approx 2$ \\
Puppy 1 & {$[12]$} & 1.5 & 0.2 & 0.17 & 0.5 & 0.13 & 2.9 \\
\hline Puppy II & {$[13]$} & 0.273 & 0.075 & 0.142 & 0.5 & 0.34 & 3.5 \\
Takuma & {$[14]$} & 0.55 & 0.1 & 0.34 & 0.03 & 0.001 & 0.09 \\
\hline MIT-Cheetah & {$[15]$} & 33 & 0.5 & 0.7 & 6 & 7.34 & 8.57 \\
MIT-Cheetah II & {$[16]$} & 33 & 0.5 & 0.7 & 4.5 & 4.13 & 6.43 \\
\hline Bobcat & {$[17]$} & 1.03 & 0.125 & 0.166 & 0.78 & 0.5 & 4.7 \\
\hline Lynx-SV1 & {$[18]$} & 1.2 & 0.154 & 0.224 & 0.75 & 0.25 & 3.3 \\
Lynx-SV2/3 & {$[18]$} & 1.2 & 0.154 & 0.226 & 0.6 & 0.24 & 2.7
\end{tabular}

a) Methods of agility-benchmarking: Agility as a term is used in a manifold of robotics papers, but often only as a synonym for forward speed, maneuverability (e.g. the ability to do controlled change in movement or direction) or is just mentioned as a term symbolizing high performance of a robot or animal [19]-[28]. Forward speed is surely a key factor in agility and thus often used for comparison of numerous robots with a large variance in size (see Table I). Well defined scores like the Froude number used by [29] or the normalization of the robots' speed to body-lengths per second (BL/s) in combination with the Cost of Transport (CoT) [30] are accepted throughout the community. In our opinion this acceptance has its basis in the ease of use of these scores. One only needs simple geometrical measurements as well as energy- and speed-data for their generation. This ensures accessibility and easy understanding for a broad audience. Both scores quantify straight forward or backward locomotion and thus are not sufficient when looking at the rich feature set of agile locomotion. Some scientists tried to tackle the issue by introducing performance matrices or set up standard environments to benchmark their robots. As an example of implementation of test areas within specific scenarios, the framework of search and rescue operations, such as "NIST 
standard Test Bed for Urban Search and Rescue," is often chosen. Hereby the primary measure is not the agility of a robot itself, but amongst others, the number of victims found in a cluttered terrain [31]-[34]. Of course, navigation through an almost realistic disaster terrain is very demanding for a robot and may even not yet be possible, but this method gives no quantitative and comparable values for the desired specific agility benchmarking. When switching from test-bench based benchmarks to more analytical ones an analysis of acceleration capabilities in the framework of dynamic capability equations can be used to identify the performance of a robot [35], [36]. This method is rather complex and thus not very attractive for us, as we aim for an easy-to-use benchmark. [37] proposed a framework for benchmarking versatility in comparison to the robots complexity. Although this approach showed many exciting ideas, a big challenge is the complexity of the method itself. It includes comparisons of land, water, and aerial robots in one framework, which makes it easy to get confused. A critical factor in their proposed method is a weighting system. The weights are chosen without clear background data. Unfortunately, this makes agreeing with and following the proposed method difficult. The last attempt on measuring agility we want to mention is shown for the case of the leaping quadruped Canid [38]. [39] introduced a coefficient for specific agility during stance using the mass-normalized change in extrinsic body energy. They argue that the change in extrinsic body energy, especially during leaping, reflects the effect of an agile movement on the robot best. This specific agility score is not dimensionless and thus scaling effects have to be taken into account when comparing different robots. Another leaping metric can be found in [40]. Here the frequency and velocity of consecutive jumps are brought into relation with each other. The metric itself resembles our approach strongly as it takes the time for the maneuver as well as the height into account. The main differences are the non-dimensionless nature of the proposed score and the fact of taking an average speed of multiple jumps instead of putting emphasis on repeatability. Positioning these approaches as a valid alternative to our method, especially when performing jumps, we acknowledge the strong influence of the robot energetics and thus will try to incorporate an inspired value, see Section IV.

b) Conclusion from agility definitions or evaluations in robotics: The realm of robotics did not yet produce common methods to define agility. Speed (non-dimensional and dimensional) is well researched, and some attempts to benchmark other tasks are made. The most used benchmarking scores are very easy to use, only requiring little experimental data and utilizing geometrical measurements of the robots to scale between platforms. The approach of setting up a test bench is also valid, but unfortunately, very time consuming, expensive and complex. Concluding from our observations, our proposed method should be easy to use, with as little experimental data as possible and incorporate robot energetics, if applicable.

\section{Proposed Method for Benchmarking Agility}

As agility, in general, is highly related to the speed of how a task can be done, all scores proposed are normalized and dimensionless speeds, guaranteeing comparability between different robots and animals. Normalization and dimensionless scores are achieved by employing the scaling method of Hof [41]. As described in the following, there are thirteen scores which should, in the authors' opinion, form the core concept of agility in legged terrestrial systems. These initial tasks were inspired by the ones generally visible in dog agility competitions as well as behaviors that enable the animals to react quickly to changes in their environment. Additional tasks can and may be added in the future to extend the benchmark. The higher the scores for turning $\left(A_{t s}\right.$ and $\left.A_{t r}\right)$, leaping $\left(A_{l}\right.$, $A_{l v}$ and $\left.A_{j}\right)$, slope running $\left(A_{s 1}, A_{s 2}\right.$ and $\left.A_{s 3}\right)$, standing up $\left(A_{s t 1}, A_{s t 2}\right)$, sidestepping $\left(A_{s s t e p}\right)$ as well as forward and backward locomotion $\left(A_{f l}\right.$ and $\left.A_{b l}\right)$ are, the better the agility is. The lowest possible score is zero. Although negative scores are possible, we disregard them as they only show how bad a system is in achieving a motion. This badnessscore may nevertheless give researchers clues for their robot improvement to reach an agility score higher than 0 . To take the quality regarding precision and repeatability into account, certain variance factors will be introduced for each score. Furthermore, an overall weighted agility score as the sum of the components is proposed and also correlated with the cost of agility (COA). The scores are kept as simple as possible (see Table II and respective description paragraphs) to allow easy experimental implementation.

To provide the needed baseline for agile locomotion, we decided to, exemplary, analyze the performance of agility dogs performing different tasks during competitions and training sessions. The baseline will serve as a reference for the agility scores, enabling fair contribution of each score to the overall agility benchmark.

1) Measurement of Geometrical Values: To allow uniformity when defining the geometrical values for robots with different shapes and number of legs, the following scheme should be applied. Robot length $l_{R}$ is to be measured from the first hip axis to the last one with fully elongated body. The width $w_{R}$ is defined as the distance between the outer edges of two opposite legs at hip level. The last value, robot height $h_{R}$ is taken as distance from ground to hip-axis in an upright standing posture (leg joints extented to reach maximum leglength). The same posture is used when defining the height of the center of mass (COM), $h_{C O M}$, also including the mass of the legs.

2) Turning with a Radius $A_{t r}$ : The core of the equation for $A_{t r}$ is represented by the time taken for the maneuver $t$ [s] and number of turns achieved in that time $p$. After Hof [41] this is implemented by division of the time through the square root of robot height $\left(h_{R}[\mathrm{~m}]\right)$ divided by gravity $(\mathrm{g}$ $\left.\left[\mathrm{m} \mathrm{s}^{-2}\right]\right)$ In addition, a term for normalization of the turning radius $r$ [m] with the robots height $h_{R}[\mathrm{~m}]$ is added. The first term is the variance of the turn $q_{t r}$ [\%]. The variance is describing how well the robot can perform an ideal circle and is measured by the distance (orthogonal to the movement direction) of starting point to endpoint after 10 consecutive turns. If the robot's deviation from the ideal circle is larger during the turn (e.g. irregular circle, ellipse etc.) and coming back to the starting point, the larger distance should be chosen 
Table II: Summary of benchmarking calculations, with scores for turning $\left(A_{t r}\right.$ and $\left.A_{t s}\right)$, leaping $\left(A_{l}, A_{l v}\right.$ and $\left.A_{j}\right)$, slope running $\left(A_{s 1}, A_{s 2}\right.$ and $\left.A_{s 3}\right)$, standing up $\left(A_{s t 1}, A_{s t 2}\right)$, sidestepping $\left(A_{\text {sstep }}\right)$ as well as forward and backward locomotion $\left(A_{f l}\right.$ and $\left.A_{b l}\right)$

\begin{tabular}{l|l} 
Calculation & Variance \\
\hline$A_{t s}=\frac{p}{t} \cdot \sqrt{\frac{h_{R}}{g}}$ & not needed \\
\hline$A_{t r}=q_{t r} \cdot \frac{h_{R}}{r} \cdot \frac{p}{t} \cdot \sqrt{\frac{h_{R}}{g}}$ & $q_{t r}=1-\left(\frac{\Delta r}{0.25 \cdot r}\right)$ \\
\hline$A_{j}=q_{j} \cdot \frac{h_{j}}{h_{R}} \cdot \frac{1}{t} \cdot \sqrt{\frac{h_{R}}{g}}$ & $q_{j}=1-\left(\frac{\Delta h_{j}}{0.25 \cdot h_{j}}\right)$ \\
$A_{l}=q_{l} \cdot \frac{l_{l}}{h_{R}} \cdot \frac{1}{t} \cdot \sqrt{\frac{h_{R}}{g}}$ & $q_{l}=1-\left(\frac{\Delta l_{l}}{0.25 \cdot l_{l}}\right)$ \\
$A_{l v}=q_{l v} \cdot \frac{l_{l v}}{h_{R}} \cdot \frac{1}{t} \cdot \sqrt{\frac{h_{R}}{g}}$ & $q_{l v}=1-\left(\frac{\Delta l_{l v}}{0.25 \cdot l_{l v}}\right)$ \\
$A_{s 1}=q_{s} \cdot i_{s 1} \cdot \frac{h_{c o m}}{h_{R}} \cdot \frac{l_{s}}{h_{R}} \cdot \frac{1}{t} \cdot \sqrt{\frac{h_{R}}{g}}$ & $q_{s}=1-\left(\frac{\Delta w_{s}}{w_{R}}\right)$ \\
$A_{s 2}=q_{s} \cdot\left(-i_{s 2}\right) \cdot \frac{h_{c o m}}{h_{R}} \cdot \frac{l_{s}}{h_{R}} \cdot \frac{1}{t} \cdot \sqrt{\frac{h_{R}}{g}}$ & $q_{s}=1-\left(\frac{\Delta w_{s}}{w_{R}}\right)$ \\
\hline$A_{s 3}=q_{s} \cdot i_{s 3} \cdot \frac{h_{c o m}}{h_{R}} \cdot \frac{h_{R}}{w_{R}} \cdot \frac{l_{s 3}}{h_{R}} \cdot \frac{1}{t} \cdot \sqrt{\frac{h_{R}}{g}}$ & $q_{s}=1-\left(\frac{\Delta w_{s}}{w_{R}}\right)$ \\
$A_{s t 1}=\phi \cdot q_{s t} \cdot \frac{1}{t} \cdot \sqrt{\frac{h_{R}}{g}}$ & $q_{s t}=\frac{m_{s u c c e s}}{10}$ \\
$A_{s t 2}=q_{s t} \cdot \frac{1}{t} \cdot \sqrt{\frac{h_{R}}{g}}$ & $q_{s t}=\frac{m_{s u c c e s}}{10}$ \\
\hline$A_{s s t e p}=q_{s s t e p} \cdot \frac{w_{s}}{h_{R}} \cdot \frac{1}{t} \cdot \sqrt{\frac{h_{R}}{g}}$ & $q_{s s t e p}=1-\frac{\Delta l_{s}}{0.25 \cdot l_{R}}$ \\
\hline$A_{f l}=q_{f l} \cdot \frac{l_{f l}}{h_{R}} \cdot \frac{1}{t} \cdot \sqrt{\frac{h_{R}}{g}}$ & $q_{f l}=1-\frac{\Delta w_{f l}}{w_{R}}$ \\
\hline$A_{b l}=q_{b l} \cdot \frac{l_{b l}}{h_{R}} \cdot \frac{1}{t} \cdot \sqrt{\frac{h_{R}}{g}}$ & $q_{b l}=1-\frac{\Delta w_{b} l}{w_{R}}$ \\
\hline
\end{tabular}

to calculate the turning variance. We define a variance larger than one quarter of the mean turning radius to mark highly unreliable behavior and thus set the agility value to zero. In case of on-the-spot turning, this value would become 1 and the radius 0 , resulting in a non-solvable equation. Thus the formula for $A_{t s}$ applies in this special case.

3) Turning on the Spot $A_{t s}: A_{t s}$ is chosen if the robot's rotational axis is exactly in the geometric center, otherwise turning with a radius applies. A robot capable of turning on the spot will achieve a $A_{t r}$ approaching infinity and thus always have the highest possible value. In consequence, to avoid such an unrealistic scenario, we decided to create a seperated score with a turning radius equal 0 . This also allows the robot to achieve higher overall agility, if the robot shows both skills. The main features of the on-the-spot turn are the time needed to complete the turning procedure $t[\mathrm{~s}]$ and the number of turns $p$ around the robot middle axis, which results in angular speed. A variance or quality of the turn is not needed as any diversion from the rotation around the middle axis results in a turning with a radius and the respective score applies. The only normalization with respect to the robot that is needed is a dimensionless time.

4) Jumping $A_{j}$ and Leaping $A_{l(v)}$ : Both movements (jumpig with $A_{j}$ and leaping $A_{l}$ as well as $A_{l v}$ ) are very agile and explosive, but pose a risk for robots as damage can occur. Successful execution is thus a sign for high capability and should be represented in our benchmark. Jumping has no or only little horizontal movement as it is describing how high the robot can jump, whereas one focuses on the horizontally traveled distance in air when talking about leaping. Both scores include the time for the maneuver $\mathrm{t}[\mathrm{s}]$, scaled dimensionless, the height of the jump $h_{j}[\mathrm{~m}]$ (measured as distance from the hip when standing and at apex height) and the length of the leap $l_{l}[\mathrm{~m}]$ (measured at the same foot at liftoff and after the leap at touchdown) normalized with the robots' hip height $h_{R}$ [m]. The variance factors $q(j)[\%], q(l)[\%]$ and $q(l v)[\%]$ give a notion of the repeatability and precision by giving the mean deviation $\Delta h_{i}[\mathrm{~m}]$ in percent of the overall mean jumping height $h_{j}$ or leaping length $l_{l}$ [m], measured from 10 repetitions. Again one quarter of the respective mean value will be the boundary of failure for an agile robot. Leaps out of a running motion should logically increase $A_{l}$ through the initial thrust. We encourage users of our benchmark to acknowledge the fact with an index at the score $A_{l v}$. This score is not different from $A_{l}$ but gives an indicator of the motion the robot was in, when the leap occurred. The initial velocity should be noted as a remark.

5) Slope Running $A_{s(1-3)}$ : Navigation on slopes (climbing would be an exceptional case where the inclined surface is at least orthogonal to the ground) needs almost the biggest variety of parameters to be defined sufficiently in the scores $A_{s(1-3)}$. Slopes up- and downwards, with the same calculation but one working with and one against gravity, as well as slopes inclined towards the sagittal plane of the robot and thus orthogonal to the movement direction should be considered. This will be implemented by setting the respective inclination $i_{s 1}, i_{s 2}$ and $i_{s 3}$ in [\%], whereas $i_{s 1}$ and $i_{s 3}$ are positive, opposing the negative $i_{s 2}$. Geometrical measurements and time are used for calculation of the score. Normalization is taken into account with the height of the robot's center of mass $h_{\text {com }}$ [m], its width $w_{R}[\mathrm{~m}]$ (especially important as robots with a wide or sprawling posture, have a strong advantage in the sideslope task due to the smaller possibility of falling to the side) and robot height $h_{R}[\mathrm{~m}]$. To receive a dimensionless speed value for the agility representation, the time for the maneuver $t[\mathrm{~s}]$ and the distance traveled $l_{s}[\mathrm{~m}]$ are scaled by the robot height $h_{R}[\mathrm{~m}]$ and gravity (as seen before). The variance of the performance influences the measure with $q_{s}$ where the percentile deviation from a straight path after a distance of 10 body-lengths in respect to the robot width is calculated. More than one robot width will be seen as too large of a variance and thus considered as not precise enough, setting the agility score to zero.

6) Standing up $A_{s t(1-2)}$ : Standing up $\left(A_{s t(1-2)}\right)$ is mostly related to the time $t[\mathrm{~s}]$ needed to get up from a crouched posture with the trunk touching the ground. This basic behavior is sometimes hard to stabilize and thus worth being considered in our benchmark as score $A_{s t 2}$. Even higher skill and agility is needed if the robot is lying on the side or even upside down, score $A_{s t 1}$. This is represented by the angle the robot's sagittal or transversal plane has in the lying position to the normal vector of the flat ground $\phi[\mathrm{rad}]$. The variance is given by the percentage of successful, stable lifts $m_{\text {success }}$ in respect to the total number of $\mathbf{1 0}$ trials. A successful lift is defined by the robot not falling over for a period of minimum $5 \mathrm{~s}$ after reaching its standard locomotion posture.

7) Sidestep (non-holonomic) $A_{\text {sstep }}$ : Moving sidewards is defined through the width of one step $w_{s}[\mathrm{~m}]$ normalized by the robot height $h_{R}[\mathrm{~m}]$ and the time needed to perform the 
maneuver $t[\mathrm{~s}]$ in its dimensionless form, leading to the score $A_{\text {sstep. }} q_{\text {sstep }}[\%]$ describes the variance of the sidestep by relating the deviation from a straight path $\Delta l_{s}[\mathrm{~m}]$ in terms of robot length $l_{R}[\mathrm{~m}]$ after 10 steps. A variance of more than a quarter of the robot length is defined as not precise and thus not agile.

8) Forward $A_{f l}$ and Backward Locomotion $A_{b l}$ : The last part of the agility scores is related to the most known locomotion type in mobile robotics, straight forward and backward locomotion $\left(A_{f l} / A_{b l}\right)$. To calculate, we need the respective distance traveled forwards $l_{f l}[\mathrm{~m}]$ and backwards $l_{b l}[\mathrm{~m}]$ normalized with the robot height $h_{R}[\mathrm{~m}]$ and the measured time of the respective movement $t[\mathrm{~s}]$ in the dimensionless form. The variance is again the deviation from a straight path with respect to robot width $w_{R}[\mathrm{~m}]$ after a distance of $\mathbf{1 0}$ body-lengths.

9) General Agility $A_{\text {gav\%: }}$ All scores describe agility in a certain manner and we encourage evaluating them separately, as the qualities of individual characteristics can be seen clearly on a direct comparison. Nevertheless, it is interesting to combine them with a global overview of the system's agility in comparison to a baseline besides looking at them separately. The weighted average agility $\left(A_{g a v} \%\right.$ of all agility-elements allows us to compare different systems which cannot perform the same tasks fairly easy. This is based on our view, that a robot should be called agile not only if it can manage to excel in one task, but also if it can execute various tasks with lower performance. The normalization with our exemplary dog-databaseline gives weight to the different scores and thus allows a fair comparison. $\mathrm{i}$ is the number of scores achieved by the robot and $\mathrm{j}$ the total number of scores available in the benchmark (needed to achieve a maximum weighted average agility of $100 \%)$.

$$
A_{\text {gav } \%}=\frac{\sum_{i} \frac{A_{i-\text { robot }}}{A_{\text {i-baseline }}} \cdot 100 \%}{j}
$$

10) Baseline Values: Even with the scores established, it remains challenging to find a good reference frame. Again, inspiration and observation from nature might help to handle this task. Dog-agility competitions are highly standardized (e.g., obstacle length and height, weight classes of the dogs) and video analysis can serve as a valuable tool for measuring time during the run of a competitor. In our case, the frames until completion of each task were counted and through the video recording frequency, the respective time was calculated. The physical parameters of the participating dogs were taken as a mean of the size-classifications in the dog-agility rulebook and by evaluating the standard measurements for the respective race. The height of the center of mass was approximated as $100 \%$ of the hip-height. This follows from [42] where the authors placed weights at the hip height, claiming it to be close to the center of mass. The size of the respective obstacle to the agility-task performed is taken from the dog-agility rulebook as well [43], [44]. All of these factors in mind, Table III shows scores that, in the author's opinion, stand for exceptional agility and can serve to norm the previously defined agility scores. Thus the values of the aggregated dog agility in
Table III represent a value of $100 \%$ for each respective agility score. Quality is always seen as the highest, meaning 1. Unfortunately, dog-agility does not cover all of the proposed agility-scores or combines them within fluid transitions which let the need for other sources arise. This concerns (1) on the spot turning, (2) leaping out of stance, (3) side slope running, (4) side-stepping (5) forward and (6) backward locomotion. On the spot turning can be found in some other video sources where dogs of different sizes perform tricks. The turn itself happens (seemingly independent of size) very fast and is thus approximated as a duration of $t=0.3[\mathrm{~s}]$.

Conclusive leaping data out of the stance is sparse and will thus be approximated as half the running-leap value. Pure side slope running is rarely noted in nature as the animal would most likely change direction to either descent or climb the slope. We hypothesize that a possible side-sloperunning cannot be performed faster than normal slope ascent. Consequently, we assign the same value. As before sidestepping rarely occurs in nature but can be seen in trick shows like Dog Dance [45]. The movement itself can be achieved and performed relatively fast after training. The measured value from [46] gives a time of $t \approx 0.2[\mathrm{~s}]$ per step with step widths of half a body width.

Table III: (left) Baselines for agility scores extracted from dog-agility competitions; mean values for 3 winners of the competition in different size classes, geometries taken as mean of the performing dogs; (right) Baselines for agility scores extracted from dog-agility competitions and merged with intuitive values to reach the 'aggregated dog' (incl. data used to calculate); length is representing the radius for the turning score, the inclination is calculated from height and length of the obstacle; h- height, l- length, w- width

\begin{tabular}{l|lll|lllll} 
& Large & Medium & Small & \multicolumn{5}{c}{ Dog Aggregated } \\
\hline $\mathrm{h}[\mathrm{m}]$ & 0.53 & 0.39 & 0.3 & \multicolumn{5}{c}{0.41} \\
$\mathrm{l}[\mathrm{m}]$ & 0.63 & 0.53 & 0.43 & & & 0.53 & \\
$\mathrm{w}[\mathrm{m}]$ & 0.35 & 0.3 & 0.25 & & & 0.3 & \\
\hline & $\mathrm{Nr}[]$ & $\mathrm{Nr}[]$ & $\mathrm{Nr}[]$ & $\mathrm{t}[\mathrm{s}]$ & $l_{o}[\mathrm{~m}]$ & $h_{o}[\mathrm{~m}]$ & $\mathrm{q}$ & $\mathrm{Nr}[]$ \\
\hline$A_{t s}$ & & & & 0.3 & & $p=1$ & 1 & 0.679 \\
$A_{t r}$ & 0.064 & 0.047 & 0.032 & 1.18 & 0.97 & $\mathrm{p}=0.5$ & 1 & 0.036 \\
$A_{j}$ & 0.397 & 0.381 & 0.394 & 0.61 & & 0.48 & 1 & 0.394 \\
$A_{l}$ & & & & 0.53 & 0.48 & 0.305 & 1 & 0.453 \\
$A_{l v}$ & 1.055 & 0.874 & 0.643 & 0.53 & 0.97 & 0.305 & 1 & 0.916 \\
$A_{s 1}$ & 0.486 & 0.484 & 0.663 & 0.9 & 2.89 & 0.914 & 1 & 0.531 \\
$A_{s 2}$ & 0.486 & 0.484 & 0.663 & 0.9 & 2.89 & 0.914 & 1 & 0.531 \\
$A_{s 3}$ & & & & 0.9 & 2.89 & 0.914 & 1 & 0.531 \\
$A_{s t 1}$ & & & & 0.5 & & $\Phi=1.57$ & 1 & 0.639 \\
$A_{s t 2}$ & & & & 0.25 & & & 1 & 0.814 \\
$A_{s s t e p}$ & & & & 0.2 & 0.175 & & 1 & 0.438 \\
$A_{\text {fl }}$ & 5.877 & 6.186 & 6.529 & 1 & 12.2 & & 1 & 6.108 \\
$A_{b l}$ & & & & 1 & 3.05 & & 1 & 1.527
\end{tabular}

Forward speed can be found from literature on animal locomotion and the specifics of different animal species. In our case, often performing dog breeds were investigated and speed-values for the respective breed (Border collie, Shetland Sheepdog, Jack Russel terrier) were used [47]. Backward locomotion is unnatural to many animals as they would rather use a fast turning motion and their forward locomotion skills in combination. Videos for dogs show, again after training or in situations with no other option, backward stepping with low to medium speeds, especially compared to forward speed [48]. In our case a representative value, approximated to be 
one quarter of the maximum forward speed, can be found in the baseline-table for the aggregated dog ( Table III).

11) Cost of Agility: As we want to be able to compare different systems with each other, it can be useful, but not necessary, to include the cost of performing the above-measured tasks. For this purpose the average power consumption of each single task $P_{\text {task }}[\mathrm{W}]$ is used. This power results from the difference of the standby power consumption and the one during the execution of the task. The robot weight $m_{R}[\mathrm{~kg}$, which strongly influences the difficulty to perform certain tasks (e.g., jumping or leaping) is an additional factor. In combination with the specific agility score $A_{\text {task }}$ we introduce a power density that can be compared between different systems. This Cost of Agility resembles the often used and well-established Cost of Transport, that was introduced to further quantify forward locomotion [30].

$$
C O A=\frac{P_{\text {task }}}{A_{\text {task }} \cdot m_{R}} \quad[\mathrm{~W} \mathrm{~kg}]
$$

The Cost of Agility cannot yet be included in the baselinevalues derived from nature as measuring metabolic cost throughout the required task execution in animals is not possible for the authors, and only insufficient data can be found in published articles [49]. Besides, this value is also directly coupled to the respective agility score, which is already standardized. We thus propose using it as-is and building a conclusive database of different cost-values over time.

12) Open Database for Agility Benchmarking: As part of this publication, we propose an online framework to enable researchers to share their experience with the agilitybenchmark. The agility-database can be found on the EPFL hosted website [agility.epfl.ch] and is open access. We hope to encourage researchers to share and compare their robot's performance to other systems in the database. Additionally, we hope developers can find new robots through this benchmark, that include features they might be interested in and thus make their innovations more efficient.

13) Experimental Setup: Due to the simplicity of the proposed method, getting good and reliable data from the experiments does not impose the need for high technology. We propose two different setups, which deliver sufficient accuracy to derive the needed parameters. Setup $\mathbf{1}$ is purely relying on a Motion capturing system (Mocap), a scale to weigh the robot and an energy measurement system (e.g. external system such as current probe on powercable combined with voltage measuremt or internal by a power-measurement IC/PCB connected to the robots battery). If a Mocap is not available it can be replaced in setup 2 with a high-speed camera and needed lighting for top and side views, and scales for heights, lenghts and angles, as described in the numbers. Having a professional motion capturing system makes recording the needed data from experiments easier. Nevertheless, it is advised, especially for illustration and comparison purposes, to record the tests with high-speed cameras. If setup $\mathbf{2}$ is chosen, a scale for the respective movement should be in the picture-frame of the camera, so the achieved movement can be quantified. The time can be extracted by counting the recorded frames and bringing them in correlation with the respective frame- rate of the recording-system or using the time stamps of the recording.

\section{FIRST EXPERIMENTAL IMPLEMENTATION}

With the agility-benchmark being defined, implementation of existing robotic systems is the logical next step. As we have a broad range of different legged robots in the Biorobotics laboratory, we can implement a proof-of-concept directly. A general comparison of the agility scores can be found in Table IV.

1) Overview over the Selected Robots: The first series of robots we applied our new benchmark to, come from the mammal-like quadruped family starting with CheetahCub (C-C) [6] with its under-actuated advanced spring loaded pantographic legs and good passive perturbation stability, then Cheetah-Cub-AL, a reviewed version of the aforementioned quadruped, and Cheetah-Cub-S, a robot with the same leg but actuated spine design for steering [50]. Another pantographdriven robot, Oncilla, closes the mammal-like starters with a high level of sensor integration (inertial measurement unit, joint-position, 3D-force-sensors in the feet) and respective closed-loop control, employing stumbling-correction, posturecontrol, and leg-extension-reflexes [51]. As a contrast to these cat- or dog-like robots, we also tested our sprawling posture robot Pleurobot. It features a highly actuated spine in combination with an extremely low COM (center of mass) as found in its biological counterpart, the salamander [52]. All robots are characterized in Figure 1 and Table IV.

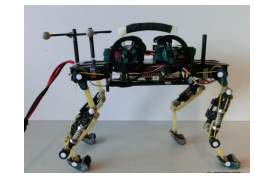

(a) Cheetah-Cub

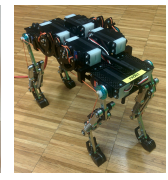

(b) C-C-AL

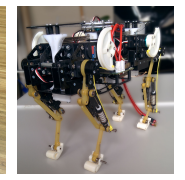

(c) C-C-S

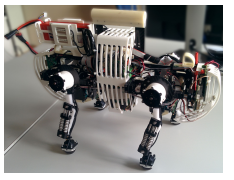

(d) Oncilla

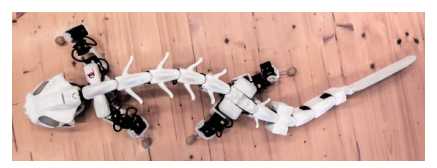

(e) Pleurobot

Figure 1: Robots, selected for an initial test of the benchmark

2) Experimental Results and Discussion: Depicted in Table IV, the robots used in the lab are far less agile (at least in the global score) than our baseline dogs (Table III). Nevertheless, the strong points of the robots become evident, and one can see the whole skill set they can use. Pleurobot and Oncilla clearly show the most skills and Cheetah-Cub the highest agility when it comes to pure speed. Turning is present in multiple of the cat robots and is a factor of 4 to 20 less than the turning scores of dogs. Oncilla shows good turning scores that start coming visibly closer to the ones of dogs. Oncilla also is the only one able to turn on the spot. This table is but a first start as visualization and proof of concept, as the searchable online database can provide even easier and more visible access to the information. This proofof-concept implementation illustrates the relatively easy use of 
our benchmark, even without generation of new experimental data. Cheetah-Cub-S, for example, does not exist anymore, but data gathered in previous experiments was easily reused in our benchmark.

Table IV: Agility scores for selected robots, the optional COA was added where data was available, as some of the robots are out of commision and already existing experimental data was used

\begin{tabular}{|c|c|c|c|c|c|c|c|c|c|c|c|c|c|c|}
\hline & Dog & \multicolumn{3}{|c|}{ Cheetah-Cub } & \multicolumn{2}{|c|}{ C-C-AL } & \multicolumn{3}{|l|}{ C-C-S } & \multicolumn{2}{|l|}{ Oncilla } & \multicolumn{3}{|c|}{ Pleurobot } \\
\hline$m_{R}[\mathrm{~g}]$ & & \multicolumn{3}{|c|}{1100} & \multicolumn{2}{|l|}{1100} & \multicolumn{3}{|l|}{1160} & \multicolumn{2}{|l|}{5050} & \multicolumn{3}{|c|}{5000} \\
\hline$h_{R}[\mathrm{~m}]$ & & \multicolumn{3}{|l|}{\begin{tabular}{|l|}
0.1 \\
\end{tabular}} & \multicolumn{2}{|l|}{0.1} & \multicolumn{3}{|l|}{0.1} & \multicolumn{2}{|l|}{0.180} & \multicolumn{3}{|l|}{0.12} \\
\hline$w_{R}[\mathrm{~m}]$ & & \multicolumn{3}{|l|}{0.1} & \multicolumn{2}{|l|}{0.1} & \multicolumn{3}{|l|}{0.105} & \multicolumn{2}{|l|}{0.245} & \multicolumn{3}{|l|}{0.38} \\
\hline$l_{R}[\mathrm{~m}]$ & & \multicolumn{3}{|l|}{0.205} & \multicolumn{2}{|l|}{0.205} & \multicolumn{3}{|l|}{0.205} & \multicolumn{2}{|l|}{0.394} & \multicolumn{3}{|l|}{0.53} \\
\hline \multirow[t]{2}{*}{ Setup } & & 1 & & & $\begin{array}{ll}1 \\
\end{array}$ & & 1 & & & 1 & & 2 & & \\
\hline & & & [\%] & {$\left[\frac{W}{k q}\right]$} & & {$[\%] \quad\left[\frac{W}{k s}\right.$} & & {$[\%] \quad\left[\frac{1}{k}\right.$} & {$\left[\frac{W}{k q}\right]$} & & [\%] $\left[\frac{\mathrm{W}}{\mathrm{kg}}\right.$ & & [\%] & {$\left[\frac{W}{k g}\right]$} \\
\hline$A_{t s}$ & 0.679 & & & & & & & & & 0.014 & 2 & & & \\
\hline$A_{t r}$ & 0.036 & 0.02 & 5 & n.A. & 0.02 & $5.6 \mathrm{n} . A$ & 0.02 & $4.3 \mathrm{n}$. & n.A. & 0.08 & 21.6 n.A. & 0.001 & 3.8 & n.A. \\
\hline$A_{j}$ & 0.394 & & & & & & & & & & & & & \\
\hline$A_{l}$ & 0.453 & & & & & & & & & & & & & \\
\hline$A_{l v}$ & 0.916 & & & & & & & & & & & & & \\
\hline$A_{s 1}$ & 0.531 & & & & & & & & & 0.007 & 1.2 n.A. & 0.04 & 7.5 & n.A. \\
\hline$A_{s 2}$ & 0.531 & & & & & & & & & 0.044 & 8.3 n.A. & 0.05 & 9.4 & n.A. \\
\hline$A_{s 3}$ & 0.531 & & & & & & & & & & & 0.048 & 9 & n.A \\
\hline$A_{s t 1}$ & 0.639 & & & & & & & & & & & & & \\
\hline$A_{s t 2}$ & 0.814 & & & & & & & & & & & & & \\
\hline$A_{\text {sstep }}$ & 0.438 & & & & & & & & & & & & & \\
\hline$A_{f l}$ & 6.108 & 1.434 & 23.5 & 94.46 & 0.687 & $11.2 \mathrm{n} \cdot \mathrm{A}$ & 0.606 & $9.9 \quad \mathrm{n}$. & n.A. & 0.474 & $\begin{array}{ll}7.8 & 41.7\end{array}$ & 0.463 & 7.6 & n.A. \\
\hline$A_{b l}$ & 1.527 & 0.404 & 26.4 & n.A. & 0.353 & $23.1 \mathrm{n} . \mathrm{A}$ & 0.303 & $19.8 \mathrm{n}$. & n.A. & 0.587 & $38.4 \quad 49.5$ & 0.459 & 30 & n.A. \\
\hline$A_{g a v \%}$ & 100 & & 4.2 & & & 3.1 & & 2.6 & & & 6.1 & & 5.2 & \\
\hline
\end{tabular}

\section{CONCLUSION AND FUtURE WORK}

Starting in the introduction, we tasked ourselves to understand the concept of agility, its definition, and quantification. In this article, we strive to give one possible answer in the form of the qualitative definition of a previously not-clearly defined robot performance trademark agility and hope to inspire more development towards a better understanding of nature and robotics. We aim at generating a better understanding of new and existing robotic systems, by putting forward means to benchmark them in more detail. Nevertheless, the acceptance of the proposed agility-benchmark is not easily predictable. We hope to generate a means for the focused development of new and agile robots, based on the found agility-qualities and their causes be it in control, electronics or mechanics of existing and benchmarked systems. The agility scores could be used as fitness functions for the optimization of mechanisms and their respective control, including learning approaches. With these main outcomes, we think of setting a high baseline for robot development in the future and bringing legged robotics one step closer to application in more realistic and challenging environments. As researchers discover and implement new robot features (such as transition capability between tasks), the agility benchmark should be extended as well, building on the open-source nature of our method. This parallel evolution of robot and benchmark will hopefully give rise to better and safer performing robots that can further our understanding of nature and one day perform in real life environments.

\section{ACKNOWLEDGMENT}

We thank Tomislav Horvat for conducting experiments with Pleurobot; Alexandre Tuleu, Mostafa Ajalloeian, Alexander
Spröwitz and Massimo Vespignani for development work done on the cat like robots; Cecilia Herbert for analyzing dog agility and other animal videos, providing additional insight and information for the development of the benchmark and Alessandro Crespi for the enormous support putting the web-database in place. The research leading to these results has received funding from the École Polytechnique Fédérale de Lausanne, the European Community's Seventh Framework Programme (FP7/2007-2013-Challenge 2-Cognitive Systems, Interaction, Robotics; grant agreement number 248311 (AMARSi)) and from the Swiss National Science Foundation through the National Centre of Competence in Research Robotics (NCCR Robotics).

\section{REFERENCES}

[1] A. Kusiak and D. W. He, "Design for agility: a scheduling perspective," Robotics and Computer-Integrated Manufacturing, vol. 14, no. 5-6, pp. 415-427, 1998. [Online]. Available: http://www.sciencedirect.com/ science/article/pii/S0736584598000179

[2] Wikipedia, "Agility." [Online]. Available: en.wikipedia.org/wiki/Agility

[3] J. Sheppard and W. Young, "Agility literature review: Classifications, training and testing," Journal of Sports Sciences, vol. 24, no. 9, pp. 919-932, 2006. [Online]. Available: http://www.tandfonline.com/doi/ abs/10.1080/02640410500457109

[4] D. Torricelli, R. S. Mizanoor, J. Gonzalez, V. Lippi, G. Hettich, L. Asslaender, M. Weckx, B. Vanderborght, S. Dosen, M. Sartori, J. Zhao, S. Schüetz, Q. Liu, T. Mergner, D. Lefeber, D. Farina, K. Berns, and J. L. Pons, "Benchmarking human-like posture and locomotion of humanoid robots: A preliminary scheme," in Lecture Notes in Computer Science (including subseries Lecture Notes in Artificial Intelligence and Lecture Notes in Bioinformatics). Springer, Cham, 2014, vol. 8608 LNAI, pp. 320-331. [Online]. Available: http://link.springer.com/10.1007/978-3-319-09435-9_28

[5] P. W. Webb, "Maneuverability - General issues," IEEE Journal of Oceanic Engineering, vol. 29, no. 3, pp. 547-555, jul 2004. [Online]. Available: http://ieeexplore.ieee.org/lpdocs/epic03/ wrapper.htm?arnumber $=1353409$

[6] A. Spröwitz, A. Tuleu, M. Vespignani, M. Ajallooeian, E. Badri, and A. J. Ijspeert, "Towards dynamic trot gait locomotion: Design, control, and experiments with Cheetah-cub, a compliant quadruped robot," International Journal of Robotics Research, vol. 32, no. 8, pp. 932-950, jul 2013. [Online]. Available: http://journals.sagepub.com/ doi/10.1177/0278364913489205

[7] I. Poulakakis, J. A. Smith, and M. Buehler, "Modeling and experiments of untethered quadrupedal running with a bounding gait: The scout II robot," International Journal of Robotics Research, vol. 24, no. 4, pp. 239-256, apr 2005.

[8] M. Raibert, "BigDog, the rough-terrain quadruped robot," in IFAC Proceedings Volumes (IFAC-PapersOnline), vol. 17, no. 1 PART 1, 2008, pp. $10823-10825$.

[9] M. Hutter, C. Gehring, D. Jud, A. Lauber, C. D. Bellicoso, V. Tsounis, J. Hwangbo, K. Bodie, P. Fankhauser, M. Bloesch, R. Diethelm, S. Bachmann, A. Melzer, and M. Hoepflinger, "ANYmal - a highly mobile and dynamic quadrupedal robot," in 2016 IEEE/RSJ International Conference on Intelligent Robots and Systems (IROS). IEEE, oct 2016, pp. 38-44. [Online]. Available: http://ieeexplore.ieee.org/document/7758092/

[10] M. Hutter, C. Gehring, M. A. Höpflinger, M. Blösch, and R. Siegwart, "Toward combining speed, efficiency, versatility, and robustness in an autonomous quadruped," IEEE Transactions on Robotics, vol. 30, no. 6, pp. 1427-1440, 2014.

[11] C. Semini, N. G. Tsagarakis, E. Guglielmino, M. Focchi, F. Cannella, and D. G. Caldwell, "Design of HyQ -A hydraulically and electrically actuated quadruped robot," Proceedings of the Institution of Mechanical Engineers. Part I: Journal of Systems and Control Engineering, vol. 225, no. 6, pp. 831-849, 2011.

[12] F. Iida and R. Pfeifer, "Cheap rapid locomotion of a quadruped robot: Self-stabilization of bounding gait," in Proceedings of the 8th International Conference on Intelligent Autonomous Systems (IAS-8), vol. 8, 2004, pp. 642-649. [Online]. Available: http: //people.csail.mit.edu/iida/papers/iida_ias8.cr.pdf 
[13] F. Iida, G. Gómez, and R. Pfeifer, "Exploiting body dynamics for controlling a running quadruped robot," in 2005 International Conference on Advanced Robotics, ICAR '05, Proceedings, vol. 2005. IEEE, 2005, pp. 229-235.

[14] T. Takuma, M. Ikeda, and T. Masuda, "Facilitating multi-modal locomotion in a quadruped robot utilizing passive oscillation of the spine structure," in IEEE/RSJ 2010 International Conference on Intelligen Robots and Systems, IROS 2010 - Conference Proceedings, 2010, pp. 4940-4945.

[15] S. Seok, A. Wang, M. Y. Chuah, D. Otten, J. Lang, and S. Kim, "Design principles for highly efficient quadrupeds and implementation on the MIT Cheetah robot," in Proceedings - IEEE International Conference on Robotics and Automation. IEEE, may 2013, pp. 3307-3312. [Online]. Available: http://ieeexplore.ieee.org/document/6631038/

[16] H. W. Park, S. Park, and S. Kim, "Variable-speed quadrupedal bounding using impulse planning: Untethered high-speed 3D Running of MIT Cheetah 2," Proceedings - IEEE International Conference on Robotics and Automation, vol. 2015-June, no. June, pp. 5163-5170, may 2015. [Online]. Available: http://ieeexplore.ieee.org/document/7139918/

[17] M. Khoramshahi, A. Sprowitz, A. Tuleu, M. N. Ahmadabadi, and A. J. Ijspeert, "Benefits of an active spine supported bounding locomotion with a small compliant quadruped robot," in Proceedings - IEEE International Conference on Robotics and Automation, 2013, pp. 33293334.

[18] P. Eckert, A. Sprowitz, H. Witte, and A. J. Ijspeert, "Comparing the effect of different spine and leg designs for a small bounding quadruped robot," in Proceedings - IEEE International Conference on Robotics and Automation, vol. 2015-June, no. June, 2015, pp. 3128-3133.

[19] D. A. Handelman, G. H. Franken, and H. Komsuoglu, "Agile and dexterous robot for inspection and EOD operations," p. 769211, 2010 [Online]. Available: http://dx.doi.org/10.1117/12.851251

[20] A. Abate, R. L. Hatton, and J. Hurst, "Passive-dynamic leg design for agile robots," 2015 IEEE International Conference on Robotics and Automation (ICRA), pp. 4519-4524, 2015. [Online]. Available: http://ieeexplore.ieee.org/document/7139825/

[21] J. Schmitt and S. Bonnono, "Dynamics and stability of lateral plane locomotion on inclines," Journal of Theoretical Biology, vol. 261, no. 4, pp. 598-609, 2009. [Online]. Available: http: //www.sciencedirect.com/science/article/pii/S0022519309003907

[22] T. Ho, S. Choi, and S. Lee, "Development of a Biomimetic Quadruped Robot," Journal of Bionic Engineering, vol. 4, no. 4, pp. 193-199, 2007. [Online]. Available: http://www.sciencedirect.com/science/article/ pii/S1672652907600328

[23] U. Saranli, M. Buehler, and D. E. Koditschek, "RHex: A simple and highly mobile hexapod robot," International Journal of Robotics Research, vol. 20, no. 7, pp. 616-631, 2001.

[24] A. T. Baisch and R. J. Wood, "Design and fabrication of the Harvard ambulatory micro-robot," Springer Tracts in Advanced Robotics, vol. 70, no. STAR, pp. 715-730, 2011

[25] A. Crespi, D. Lachat, A. Pasquier, and A. J. Ijspeert, "Controlling swimming and crawling in a fish robot using a central pattern generator," Autonomous Robots, vol. 25, no. 1-2, pp. 3-13, 2008 [Online]. Available: http://dx.doi.org/10.1007/s10514-007-9071-6

[26] Y. Li, Y. Wang, J. Geoffrey Chase, J. Mattila, H. Myung, and O. Sawodny, "Survey and introduction to the focused section on mechatronics for sustainable and resilient civil infrastructure," IEEE/ASME Transactions on Mechatronics, vol. 18, no. 6, pp. 1637-1646, 2013.

[27] J. D. Madden, "Mobile robots: Motor challenges and materials solutions," Science, vol. 318, no. 5853, pp. 1094-1097, 2007. [Online]. Available: http://www.sciencemag.org/content/318/5853/1094.abstract

[28] C. Gehring, S. Coros, M. Hutter, M. Bloesch, P. Fankhauser, M. A. Hoepflinger, and R. Siegwart, "Towards automatic discovery of agile gaits for quadrupedal robots," in Proceedings - IEEE International Conference on Robotics and Automation. IEEE, may 2014, pp. 4243-4248. [Online]. Available: http://ieeexplore.ieee.org/ lpdocs/epic03/wrapper.htm?arnumber $=6907476$

[29] R. M. Alexander, "The Gaits of Bipedal and Quadrupedal Animals," The International Journal of Robotics Research, vol. 3, no. 2, pp 49-59, jun 1984. [Online]. Available: http://ijr.sagepub.com/cgi/doi/ $10.1177 / 027836498400300205$

[30] A. D. Kuo, "Choosing your steps carefully," IEEE Robotics and Automation Magazine, vol. 14, no. 2, pp. 18-29, 2007.

[31] A. Jacoff, E. Messina, B. Weiss, S. Tadokoro, and Y. Nakagawa, "Test arenas and performance metrics for urban search and rescue robots," in Proceedings 2003 IEEE/RSJ International Conference on Intelligent Robots and Systems (IROS 2003) (Cat. No.03CH37453), vol. 3, no. November 2003, 2014, pp. 3396-3403. [Online]. Available: http://ieeexplore.ieee.org/document/1249681/

[32] A. Jacoff, B. Weiss, and E. Messina, "Evolution of a performance metric for urban search and rescue robots (2003)," DTIC Document, Tech. Rep., 2003. [Online]. Available: http://oai.dtic.mil/oai/oai?verb= getRecord\&metadataPrefix $=$ html\&identifier=ADA510436

[33] A. Jacoff, E. Messina, and J. Evans, "Performance evaluation of autonomous mobile robots," Industrial Robot: An International Journal, vol. 29, no. 3, pp. 259-267, 2002. [Online]. Available: http://www.emeraldinsight.com/doi/10.1108/01439910210425568

[34] a. Jacoff, E. Messina, J. Evans, and Others, "Experiences in deploying test arenas for autonomous mobile robots." NIST special publication, vol. 1, pp. 87-94, 2002

[35] A. Bowling, "Mobility and dynamic performance of legged robots," in Proceedings - IEEE International Conference on Robotics and Automation, vol. 2005, 2005, pp. 4100-4107.

[36] A. Bowling and S.-C. Teng, "Performance measures of agility for mobile robots," in Proceedings of the 9th Workshop on Performance Metrics for Intelligent Systems - PerMIS '09, ser. PerMIS '09. New York, NY, USA: ACM, 2009, p. 188. [Online]. Available: http://portal.acm.org/citation.cfm?doid=1865909.1865949

[37] C. Nie, X. Pacheco Corcho, and M. Spenko, "Robots on the move: Versatility and complexity in mobile robot locomotion," IEEE Robotic and Automation Magazine, vol. 20, no. 4, pp. 72-82, dec 2013 [Online]. Available: http://ieeexplore.ieee.org/document/6582554/

[38] J. L. Pusey, J. M. Duperret, G. C. Haynes, R. Knopf, and D. E. Koditschek, "Free-standing leaping experiments with a power-autonomous elastic-spined quadruped," SPIE Defense, Security, and Sensing, vol. 8741, p. 87410W, 2013. [Online]. Available: http://proceedings.spiedigitallibrary.org/ proceeding.aspx?doi=10.1117/12.2016073

[39] J. M. Duperret, G. D. Kenneally, J. L. Pusey, and D. E. Koditschek, "Towards a comparative measure of legged agility," in Springer Tracts in Advanced Robotics. Springer International Publishing, 2016, vol. 109, pp. 3-16. [Online]. Available: http: //link.springer.com/10.1007/978-3-319-23778-7_1

[40] D. W. Haldane, M. M. Plecnik, J. K. Yim, and R. S. Fearing, "Robotic vertical jumping agility via series-elastic power modulation," Science Robotics, vol. 1, no. 1, p. eaag2048, dec 2016. [Online]. Available: http://robotics.sciencemag.org/lookup/doi/10.1126/scirobotics.aag2048

[41] A. L. Hof, "Scaling gait data to body size," Gait and Posture, vol. 4, no. 3, pp. 222-223, may 1996. [Online]. Available: http://linkinghub.elsevier.com/retrieve/pii/0966636295010572

[42] D. V. Lee, "Effects of mass distribution on the mechanics of level trotting in dogs," Journal of Experimental Biology, vol 207, no. 10, pp. 1715-1728, apr 2004. [Online]. Available: http: //jeb.biologists.org/cgi/doi/10.1242/jeb.00947

[43] A. Shows, F. Competitions, B. Trials, and F. Trials, "General Regulations for Agility," Tech. Rep.

[44] T. Kennel Club, "Judges ' Guide to Agility Equipment," 2014

[45] "Dogdance." [Online]. Available: http://www.dogdance.info/en

[46] "Sidestep Youtube." [Online]. Available: www.youtube.com/watch?v= qHIRM9bcU8k

[47] "Dog-breeds." [Online]. Available: www.dogs.petbreeds.com/stories/ 5470/fastest-dog-breeds\#Intro

[48] "Backward speed Youtube." [Online]. Available: www.youtube.com/ watch? $\mathrm{v}=814 \mathrm{Gj} 0 \mathrm{MO} 6 \mathrm{Gk}$

[49] N. C. Heglund and C. R. Taylor, "Speed, stride frequency and energy cost per stride: how do they change with body size and gait?" The Journal of experimental biology, vol. 138, no. 1, pp. 301-318, 1988

[50] K. Weinmeister, P. Eckert, H. Witte, and A.-J. Ijspeert, "Cheetahcub-S: Steering of a quadruped robot using trunk motion," in 2015 IEEE International Symposium on Safety, Security, and Rescue Robotics (SSRR), 2015, pp. 1-6. [Online]. Available: http: //ieeexplore.ieee.org/lpdocs/epic03/wrapper.htm?arnumber $=7443021$

[51] A. T. Sprowitz, A. Tuleu, M. Ajaoolleian, M. Vespignani, R. Moeckel, P. Eckert, M. D'Haene, J. Degrave, A. Nordmann, B. Schrauwen, J. Steil, and A. Ijspeert, "Oncilla robot: a versatile open-source quadruped research robot with compliant pantograph legs," Frontiers in Robotics and AI, vol. 5, p. 67, 2018. [Online]. Available: https://www.frontiersin.org/articles/10.3389/frobt.2018.00067/abstract

[52] K. Karakasiliotis, R. Thandiackal, K. Melo, T. Horvat, N. K Mahabadi, S. Tsitkov, J. M. Cabelguen, and A. J. Ijspeert, "From cineradiography to biorobots: an approach for designing robots to emulate and study animal locomotion," Journal of The Royal Society Interface, vol. 13, no 119 , p. 20151089, jun 2016. [Online]. Available: http://rsif.royalsocietypublishing.org/lookup/doi/10.1098/rsif.2015.1089 
